\title{
The clinical presentation and diagnosis of ketamine- associated urinary tract dysfunction in Singapore
}

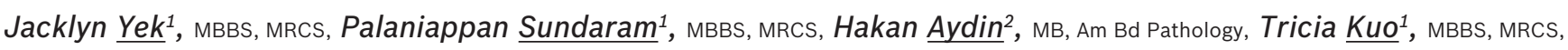
Lay Guat $\underline{\mathrm{Ng}}^{1}$, MBBS, FRCS

\begin{abstract}
Ketamine is a short-acting anaesthetic agent that has gained popularity as a 'club drug' due to its hallucinogenic effects. Substance abuse should be considered in young adult patients who present with severe debilitating symptoms such as lower urinary tract symptoms, even though the use of controlled substances is rare in Singapore. Although the natural history of disease varies from person to person, a relationship between symptom severity and frequency/dosage of abuse has been established. It is important to be aware of this condition and have a high degree of clinical suspicion to enable early diagnosis and immediate initiation of multidisciplinary and holistic treatment. A delayed diagnosis can lead to irreversible pathological changes and increased morbidity among ketamine abusers.
\end{abstract}

Keywords: bladder dysfunction, ketamin, lower urinary tract dysfunction, ulcerative cystitis

\section{INTRODUCTION}

Ketamine is an $\mathrm{N}$-methyl-D-aspartic acid receptor antagonist that is used as a short-acting anaesthetic agent. It has gained popularity as a 'club drug' due to its hallucinogenic effects. Ketamine, also known by its street names ' $\mathrm{K}^{\prime}$, 'Special K', 'Super K', 'Vitamin K' and 'Kit Kat', is sold on the streets in a white crystalline powder or tablet formulation and in an injectable prescription formulation in the clinical setting. It can be ingested, smoked, snorted, or injected intravenously or intramuscularly.

Ketamine has a progressive effect on the user. First, the abuser experiences euphoria, followed by a state of dreamy intoxication, out-of-body experiences, altered sensations and amnesia. Although these addictive effects typically last an hour or less, ${ }^{(1,2)}$ the adverse effects can be long-lasting. Commonly known adverse effects include cardiorespiratory effects, nausea, vomiting, hallucinations and convulsions. ${ }^{(3,4)}$ A less commonly elicited adverse effect is lower urinary tract symptoms (LUTS), which was identified and documented only in the last few years. Studies estimate that $20 \%-30 \%$ of ketamine abusers suffer from LUTS. ${ }^{(1,5)}$ A relationship between symptom severity and frequency/ dosage of abuse has been established, and is usually determined by the chronicity of the abuse. ${ }^{(1)}$

\section{CLINICAL PRESENTATION AND INVESTIGATIONS}

We herein illustrate the clinical presentation of four patients (three male and one female aged 21-33 years) who presented with severe intractable LUTS following ketamine abuse. The presenting complaints are shown in Table I. Gross haematuria, if present, was associated with the histological finding of ulcerative cystitis. Patients were noted to present late, with the duration of symptoms before presentation at around 2.5-10.0 years.

Routine investigations, such as urinalysis and urine culture, typically show sterile urine and occasionally, sterile pyuria, ${ }^{(1)}$ as is the case with all four patients. Early morning urine samples showed negative acid-fast bacilli smear and tuberculosis culture. Given the clinical progression of ketamine-induced cystitis (KC), we suggest an initial assessment of renal function and subsequent follow-up. ${ }^{(2,6)}$ It is believed that on cessation of ketamine abuse, the natural history of disease is not only dependent on the severity and duration of ketamine abuse but also varies from person to person. Approximately one-third of patients have resolved symptoms, one-third continue to have persistent symptoms and one-third experience worsening symptoms. ${ }^{(1)}$ Deterioration in renal function mandates more aggressive treatment.

A voiding diary should ideally be kept, as it allows objective quantification of small bladder capacity and frequent voiding. ${ }^{(6)}$ Ultrasonography of the upper urinary tract may reveal unilateral or bilateral hydronephrosis, circumferential mural thickening with a distended bladder and small bladder capacity (Fig. 1). ${ }^{(6)}$ In severe cases, there may also be upper tract involvement with ureteral wall thickening and ureteral stricture causing hydronephrosis (Figs. 2 \& 3). Bilateral hydronephrosis may be seen if both the vesicoureteric junctions are involved. Video urodynamic testing typically shows bladder capacities $<150 \mathrm{~mL}^{(5,7)}$ and decreased bladder compliance associated with detrusor overactivity (Fig. 4), complicated by vesicoureteral reflux, as seen in two of our patients (Fig. 5).

Cystoscopic examination is commonly performed under general anaesthesia, as patients are typically unable to tolerate the intense urgency and bladder pain. ${ }^{(6)}$ Findings may be consistent with ulcerative cystitis, revealing inflammation of the urothelial epithelium with glomerulations (described as small discrete submucosal petechial haemorrhages), erythematous congestion, neovascularisation and ulceration (Fig. 6). Bladder biopsy in three of our patients showed marked urothelial denudation with congestion, oedema and occasional mast 
Table I. Overview of clinical presentation of the four patients.

\begin{tabular}{lcll}
\hline Patient no. & Age $(\mathbf{y r})$ & Gender & Presentation \\
\hline 1 & 21 & Male & $\begin{array}{l}\text { Suprapubic pain, dysuria, gross haematuria, frequency } \\
\text { Frequency, urgency, hesitancy with small volume voids; } \\
\text { previous left ureteric reimplantation }\end{array}$ \\
3 & 27 & Male & Suprapubic pain, dysuria, gross haematuria, frequency \\
4 & 30 & Male & Frequency, nocturia, dysuria, suprapubic pain with filling
\end{tabular}
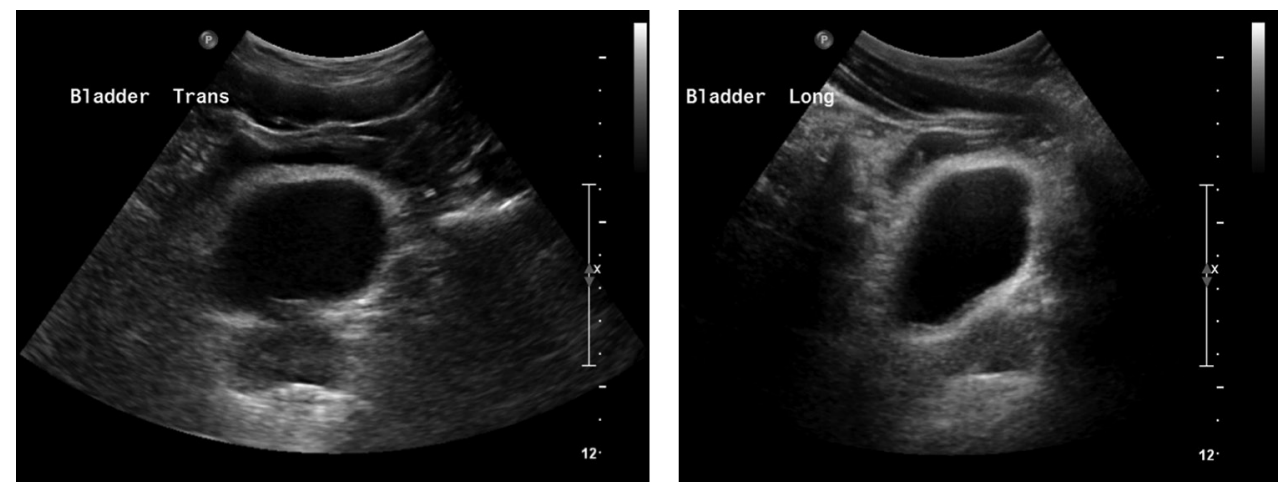

Fig. 1 US images of the bladder show circumferential mural thickening of the bladder in patient 3.
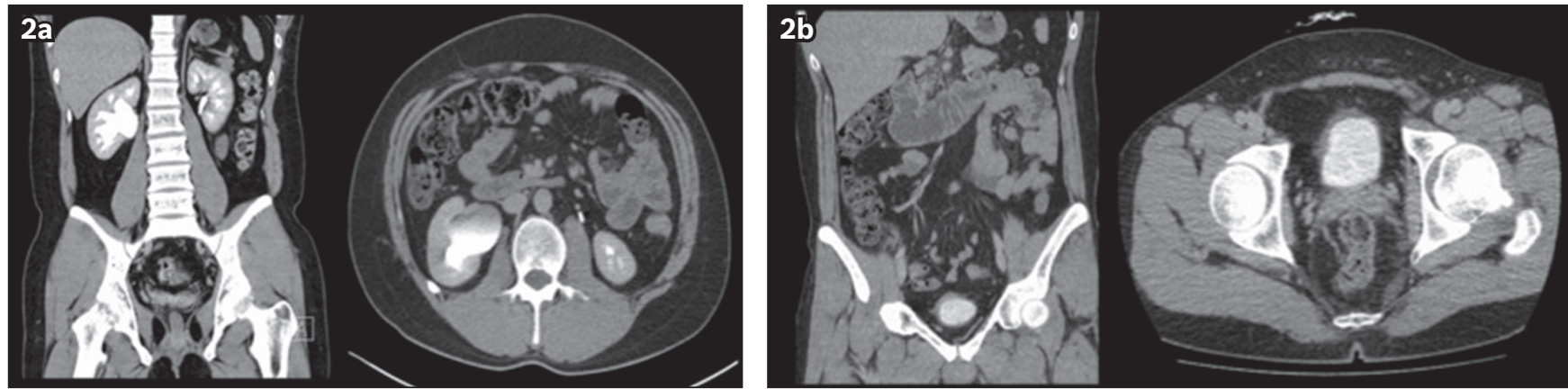

Fig. 2 CT urography image shows (a) gross hydronephrosis of the right kidney and (b) a small shrunken bladder with diffuse wall thickening and perivesical fat stranding in patient 3 .
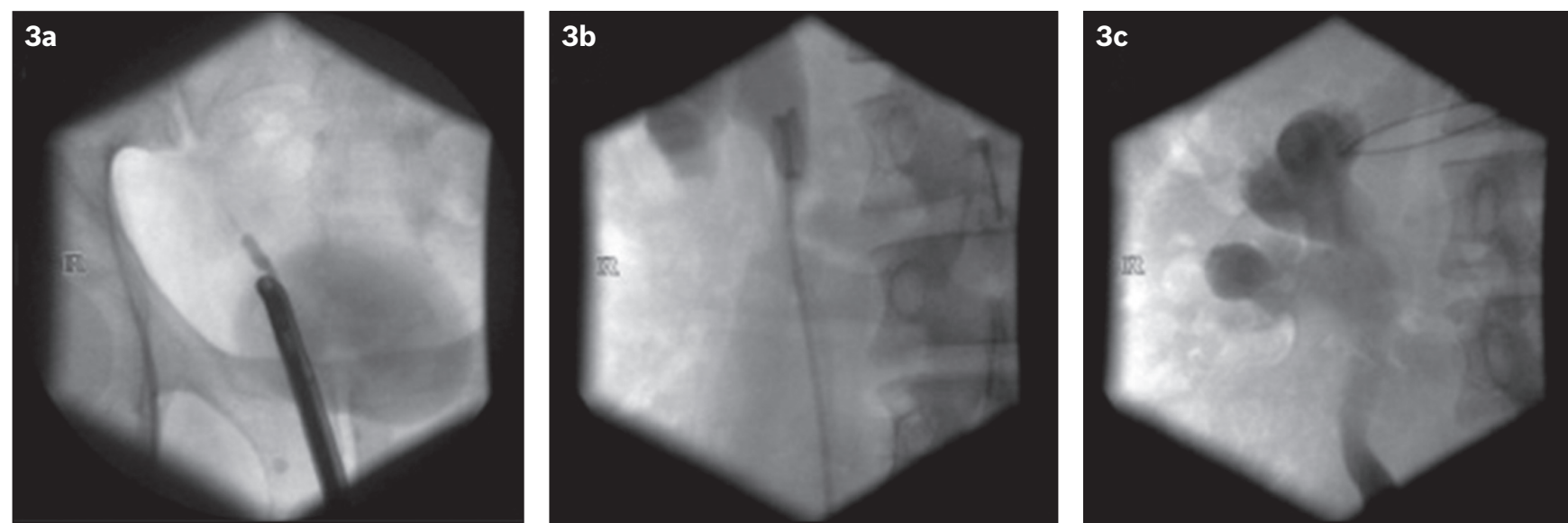

Fig. 3 Fluoroscopy images show (a \& b) retrograde double-J stent insertion and (c) gross hydronephrosis of the right kidney in patient 2.

cells in lamina propria (Fig. 7). Radiological investigations assist in the exclusion of other diagnoses, identification of complications in the upper urinary tract and surveillance of disease progression. Correlation of clinical presentation with radiological and histological findings is essential to confirm diagnosis.

\section{DISCUSSION}

\section{Pathogenesis of ketamine-induced cystitis}

Ketamine, norketamine and hydroxynorketamine are found in large quantities in the urine of ketamine abusers. ${ }^{(2)}$ Although the exact pathogenesis of $\mathrm{KC}$ remains unknown, several mechanisms have been postulated by Chu et al. ${ }^{(5)}$ Firstly, the direct toxic 


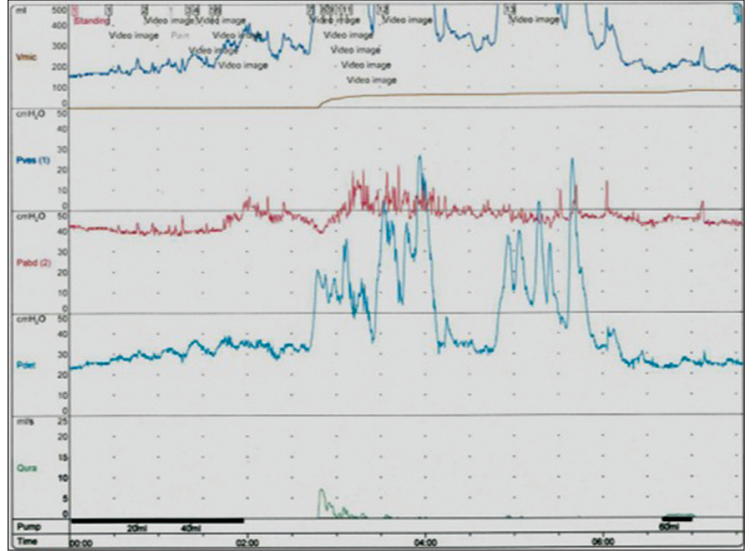

Fig. 4 Video urodynamic study (pressure-flow against time plot) shows small bladder capacity $(<100 \mathrm{~mL})$, with decreased bladder compliance associated with detrusor overactivity in patient 2 .

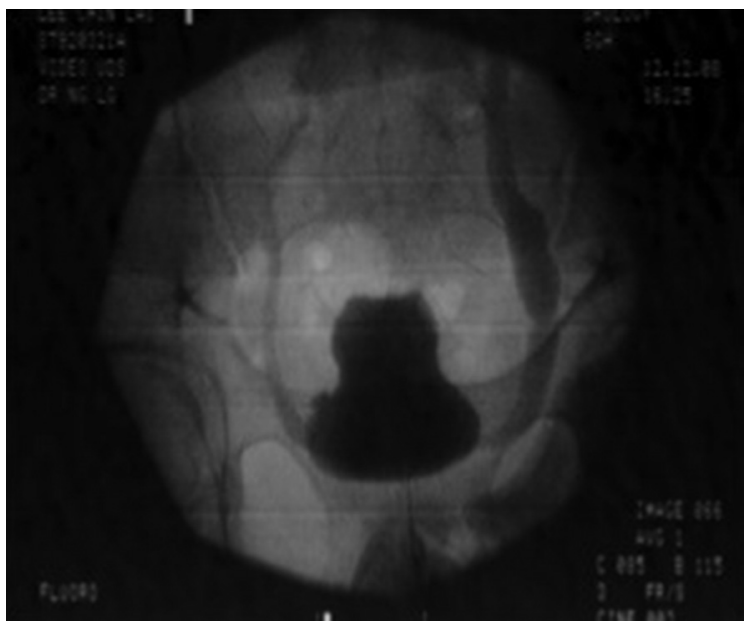

Fig. 5 Fluoroscopy image shows detrusor overactivity with vesicoureteral reflux in patient 3 .

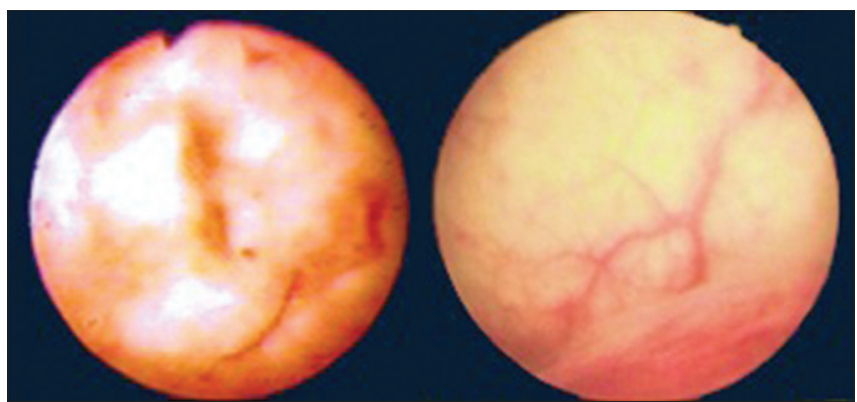

Fig. 6 Cystoscopy image shows inflammation of the urothelial epithelium with glomerulations, erythematous congestion and neovascularisation.

effect of ketamine and its metabolites on interstitial cells of the bladder and renal papillae causes a chronic inflammatory response. In the bladder, this results in submucosal and muscle fibrosis, accounting for dysuria and a contracted bladder. Similarly, in the kidney, this may result in interstitial fibrosis and structural damage, eventually leading to chronic renal insufficiency. Secondly, endothelial injury of microvessels in the urinary system leads to either compromised intrinsic microcirculation or decreased microvascular density in the subendothelium. This could potentially cause bladder ischaemia and subsequently, suprapubic pain and dysuria during bladder
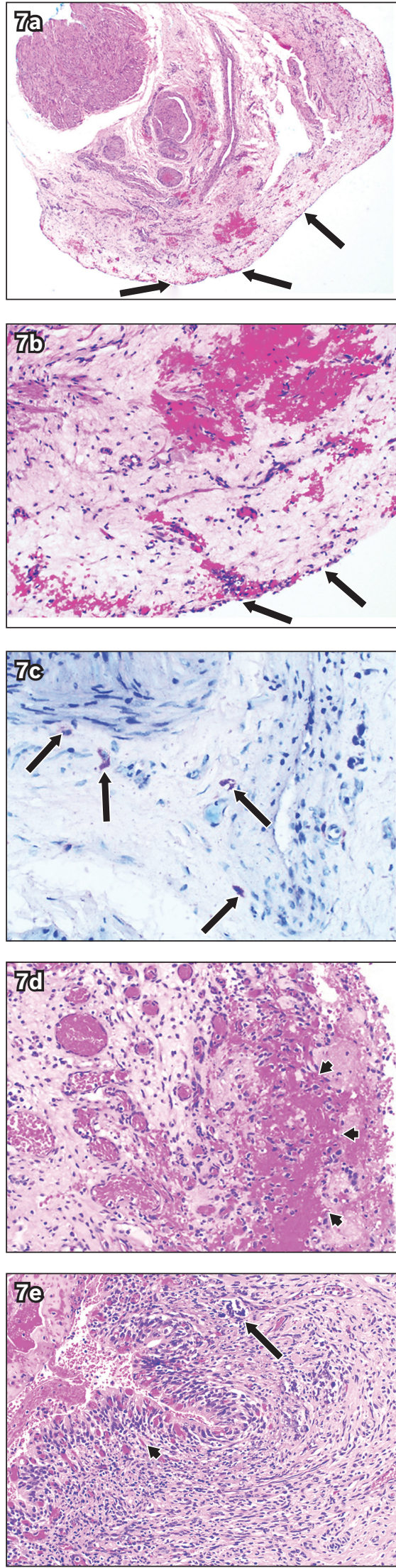

Fig. 7 Photomicrographs of the bladder tissue show ( $a \& b)$ denudation of the urothelial epithelium (arrows) with oedema and congestion in the lamina propria (Haematoxylin \& eosin, $\times 40$ and $\times 200$, respectively); (c) toluidine blue-stained scattered mast cells in the lamina propria, characterised by purple-stained cytoplasmic granules (arrows) (Toluidine blue, $\times 400)$; and ( $d \&$ e) denudation of the urothelial epithelium with adherent haemorrhage and fibrin (arrowheads) and scattered mast cells (arrow) (Haematoxylin \& eosin, × 200). 
Table II. Comparison of the characteristics of ketamine-induced cystitis and interstitial cystitis/bladder pain symdrome (BPS).

\begin{tabular}{|c|c|c|}
\hline Characteristic & Interstitial cystitis/BPS & Ketamine-induced cystitis \\
\hline Age (yr) & $\geq 40$ & $20-30$ \\
\hline Gender & Female vs. male 10:1 & Female $<$ male \\
\hline Risk factor & Previous pelvic surgery & No identified risk factors \\
\hline Associations & Chronic pain disorders & Psychosocial problems \\
\hline Symptoms & $\begin{array}{l}\text { - Suprapubic pain (including suprapubic discomfort and } \\
\text { pressure) with bladder filling that may diminish during voiding } \\
\text { - Lower urinary tract symptoms (frequency, urgency, nocturia) } \\
\text { - Dyspareunia }\end{array}$ & $\begin{array}{l}\text { - Lower urinary tract symptoms } \\
\text { (frequency, urgency, nocturia, dysuria) } \\
\text { - Suprapubic pain }\end{array}$ \\
\hline Cystoscopy & $\begin{array}{l}\text { - Hunners' ulcers }(5 \%-10 \%) \\
\text { - Glomerulations on hydrodistention }\end{array}$ & $\begin{array}{l}\text { - Neovascularisation } \\
\text { - Glomerulations }\end{array}$ \\
\hline Histopathology & $\begin{array}{l}\text { - Submucosal inflammation with denuded epithelium } \\
\text { - Mast cell activity }\end{array}$ & $\begin{array}{l}\text { - Submucosal inflammation with denuded epithelium, } \\
\text { submucosal oedema, vascular ectasia, fibrosis } \\
\text { - Detrusor inflammation and fibrosis } \\
\text { - Mast cell activity }\end{array}$ \\
\hline VUDS & $\begin{array}{l}\text { - No consistent findings } \\
\text { - Non-neurogenic detrusor overactivity }(12 \%-20 \%)\end{array}$ & $\begin{array}{l}\text { - Vesicoureteric reflux } \\
\text { - Non-neurogenic detrusor overactivity }\end{array}$ \\
\hline US of KUB & NA & $\begin{array}{l}\text { - Thickened bladder wall } \\
\text { - Hydroureteronephrosis }\end{array}$ \\
\hline Disease course & Waxing and waning & $\begin{array}{l}\text { Progressive: obstructive uropathy; } \\
\text { hydroureteronephrosis; small, contracted bladder }\end{array}$ \\
\hline
\end{tabular}

KUB: kidneys, ureter and bladder; NA: not applicable; US: ultrasonography; VUDS: video urodynamic study

filling. Microangiopathy in the renal medulla and the subsequent hypoperfusion of the papilla could then result in tubulointerstitial nephropathy and cortical atrophy of the kidney. Thirdly, urinary ketamine and its metabolites may trigger an autoimmune reaction of the bladder's urothelial epithelium and submucosa, causing an inflammatory response and scarring, resulting in a poorly compliant, small-capacity bladder.

\section{Comparison of ketamine-induced cystitis and interstitial cystitis}

Interstitial cystitis (IC) and KC share some similar features in terms of clinical presentation and histological findings. One of these features is bladder pain syndrome (BPS), which is defined by the International Continence Society as "the complaint of suprapubic pain related to bladder filling, accompanied by other symptoms such as increased daytime and night-time frequency, in the absence of proven urinary infection or other obvious pathology." ${ }^{\prime(8)}$ IC is defined as BPS with typical cystoscopic and histologic features; ${ }^{(8)}$ it is most commonly diagnosed in patients over the age of 40 years, with a female predilection of 10:1. It is associated with a high rate of prior pelvic surgery and other systemic chronic pain disorders, such as irritable bowel syndrome, chronic fatigue syndrome and fibromyalgia. However, due to the different pathophysiology of $\mathrm{KC}$ and IC, they each have a markedly different natural history of progression. $\mathrm{KC}$ affects both the upper and lower urinary tracts, leading to a small, contracted bladder and obstructive uropathy, whereas IC is a chronic bladder condition that follows a waxing and waning course. ${ }^{(8)}$ Table II compares the characteristics of the two types of cystitis.

\section{Treatment}

Ketamine detoxification is paramount in the treatment of ketamine abuse. However, the effectiveness of this treatment is dependent on the severity and duration of ketamine abuse. ${ }^{(1)}$ Although early recognition and diagnosis may prevent irreversible pathological changes, ${ }^{(6)}$ there is no single definitive management pathway. The key to effective treatment is having an individualised approach with an emphasis on compliance.

Pharmacotherapy for KC is similar to that for IC. Pain modulators such as tricyclic antidepressants (e.g. amitriptyline) act by inhibiting the presynaptic reuptake of serotonin and noradrenaline, hence modulating the transmission of nociceptive stimuli. Anti-inflammatories such as $\mathrm{H} 2$ receptor antagonists (e.g. cimetidine) act as competitive inhibitors of histamine, which is the key mediator of mast cells. Other therapeutic strategies involve approaches aimed at repairing the damaged urothelial epithelium. Pentosan polysulfate (Elmiron ${ }^{\circledR}$ ), a low-molecular-weight, heparinlike compound, acts by coating the bladder lining and repairing the glycosaminoglycan layer of the damaged urothelial epithelium. Intravesical instillations of pentosan polysulfate and hyaluronic acid have been used, as they are thought to function as a protective barrier on the urothelial epithelium.

Surgery is indicated in cases where conservative methods have failed. Intravesical botulinum toxin A injection and cystodistension have been used to increase functional bladder capacity and reduce pain. Percutaneous nephrostomy or retrograde double-J stent insertion has been performed to alleviate hydronephrosis and deteriorating renal function. Subtotal cystectomy and augmentation cystoplasty have also been performed in refractory cases to improve renal function and quality of life.

Lastly, a multidisciplinary approach involving medical social workers, psychiatrists, continence/urology nurse-clinicians and urologists should be established as many of these patients have additional psychosocial problems in addition to ketamine dependence. A holistic approach would be more effective in initiating and sustaining ketamine cessation. 


\section{Local context}

The Central Narcotics Bureau in Singapore noted that in 2013, $4.27 \mathrm{~kg}$ of ketamine was seized. This was up $10 \%$ from 2012, when $3.89 \mathrm{~kg}$ was seized. ${ }^{(9)}$ As a fear of criminal prosecution prevents patients from volunteering a history of substance abuse, directed questions are essential. Although substance abuse is uncommon in Singapore, ketamine-associated urinary tract dysfunction should be considered in young adults presenting with severe intractable LUTS.

\section{CONCLUSION}

The history of ketamine abuse should be specifically elicited. Delayed diagnosis can lead to irreversible pathological changes and increased morbidity; hence, early urology referral is advocated.

\section{REFERENCES}

1. Winstock AR, Mitcheson L, Gillatt DA, Cottrell AM. The prevalence and natural history of urinary symptoms among recreational ketamine users.
BJU Int 2012; 110:1762-6.

2. Shahani R, Streutker C, Dickson B, Stewart RJ. Ketamine-associated ulcerative cystitis: a new clinical entity. Urology 2007; 69:810-2.

3. Muetzelfeltdt L, Kamboj SK, Rees $\mathrm{H}$, et al. Journey through the K-hole: phenomenological aspects of ketamine use. Drug Alcohol Depend 2008; 95:219-29.

4. Smith KM, Larive LL, Romanelli F. Club drugs: methylenedioxymethamphetamine, flunitrazepam, ketamine hydrochloride, and gammahydroxybutyrate. Am J Health Syst Pharm 2002; 59:1067-76.

5. Chu PS, Ma WK, Wong SC, et al. The destruction of the lower urinary tract by ketamine abuse: a new syndrome? BJU Int 2008; 102:1616-22.

6. Middela S, Pearce I. Ketamine-induced vesicopathy: a literature review. Int J Clin Pract 2011; 65:27-30.

7. Tsai TH, Cha TL, Lin CM, et al. Ketamine-associated bladder dysfunction. Int J Urol 2009; 16:826-9.

8. Hanno PM, Burks DA, Clemens JQ, et al. Diagnosis and treatment of interstitial cystitis/bladder pain syndrome, amended 2014. In: American Urological Association [online]. Available at: https://www.auanet.org/ education/guidelines/ic-bladder-pain-syndrome.cfm. [Date of Access: 5 November 2014]

9. Lee $\mathrm{CL}$, Jiang $\mathrm{YH}$, Kuo HC. Increased apoptosis and suburothelial inflammation in patients with ketamine-related cystitis: a comparison with non-ulcerative interstitial cystitis and controls. BJU Int 2013; 112:1156-62. 


\section{SINGAPORE MEDICAL COUNCIL CATEGORY 3B CME PROGRAMME} (Code SMJ 201512B)

Question 1. Signs and symptoms of ketamine-induced cystitis include:

(a) Lower urinary tract symptoms such as dysuria, frequency and urgency.

(b) Gross haematuria.

(c) Fever, chills and rigors.

(d) Suprapubic pain on bladder filling.

Question 2. A 31-year-old, male chronic smoker of 20 pack-years with a history of ketamine ingestion presents with intractable urinary tract symptoms. The investigations include:

(a) Serum creatinine and estimation of renal function.

(b) Early morning urine to test for acid-fast bacilli smear and tuberculosis culture.

(c) Ultrasonography of the kidneys, ureter and bladder.

(d) Urinalysis and urine culture.

Question 3. The same patient then undergoes computed tomography urography, which reveals right hydronephrosis with upper ureteric stricture and a small contracted bladder. Are the following statements true or false:

(a) Ketamine-induced cystitis $(\mathrm{KC})$ is unlikely to be the diagnosis if patient has stopped ketamine for more than a year.

(b) Percutaneous nephrostomy may be indicated in obstructive hydronephrosis.

(c) Clinical presentation and radiological findings alone are sufficient to clinch a diagnosis of ketamineinduced cystitis.

(d) The direct toxic effect of ketamine and its metabolites can account for the pathogenesis of obstructive uropathy.

Question 4. Regarding the differences between ketamine-induced cystitis (KC) and interstitial cystitis (IC):

(a) Clinical progression of $\mathrm{KC}$ often waxes and wanes.

(b) $\mathrm{KC}$ is often associated with systemic chronic pain disorders, such as fibromyalgia, chronic pain syndrome and irritable bowel syndrome.

(c) Hunner's ulcers are always seen on cystoscopy in patients with IC.

(d) IC affects women more than men in a ratio of 10:1.

Question 5. Treatment of ketamine-induced cystitis includes:

(a) Pentosan polysulfate acts by coating the bladder lining and repairing the glycosaminoglycan layer of the damaged urothelial epithelium.

(b) Pharmacotherapy of KC is similar to that of IC.

(c) A multidisciplinary team of medical social workers, psychiatrists and urology nurse clinicians should be involved.

(d) Augmentation cystoplasty is indicated in all patients with frequency and small bladder volumes.

True

False

$\square$

$\square$

\section{Doctor's particulars:}

Name in full

MCR number

Email address

$$
\text { : }
$$
Specialty:

\section{SUBMISSION INSTRUCTIONS:}

(1) Log on at the SMJ website: http://www.sma.org.sg/publications/smjcurrentissue.aspx and select the appropriate set of questions. (2) Provide your name, email address and MCR number. (3) Select your answers and click "Submit".

RESULTS:

(1) Answers will be published in the SMJ February 2016 issue. (2) The MCR numbers of successful candidates will be posted online at the SMJ website by 1 February 2016. (3) Passing mark is $60 \%$. No mark will be deducted for incorrect answers. (4) The SMJ editorial office will submit the list of successful candidates to the Singapore Medical Council. (5) One CME point is awarded for successful candidates.

Deadline for submission: (December 2015 SMJ 3B CME programme): 12 noon, 25 January 2016. 